\title{
Avaliação da resistência adesiva da recolagem de bráquetes ortodônticos após diferentes protocolos de remoção de resina residual
}

\author{
Evaluation of bond strength of orthodontic brackets after rebonding of different protocols \\ removal of residual resin
}

Edson D’Agnoluzzo Filho ${ }^{1}$

Welton Bohry de Souza ${ }^{1}$

Haroldo Amorim de Almeida ${ }^{2}$

Ana Maria Martins Brandão ${ }^{2}$

Gustavo Antonio Martins Brandão ${ }^{2}$

\section{RESUMO}

A seleção adequada do instrumental é fundamental para uma eficiente remoção de resina residual do esmalte, o que pode influenciar de maneira positiva na resistência adesiva após recolagem de bráquetes ortodônticos. Para avaliar diferentes protocolos de remoção de resina residual do esmalte dental, visando a manutenção de uma resistência adesiva adequada na recolagem de bráquetes ortodônticos, foram utilizados 40 incisivos bovinos, divididos em 4 grupos (G1, G2, G3 e G4), com 10 dentes cada. Foi confeccionada uma matriz de resina, após condicionamento com ácido fosfórico em cada espécime, para simular o adesivo remanescente no esmalte dental. Em seguida, foi feita a remoção deste remanescente conforme os protocolos propostos. Os dentes do G1 foram trabalhados com ponta CVD uniangulada, os do G2 com broca carbide baixa rotação, os do G3 com broca carbide alta rotação e os do G4 com ponta diamantada. Após essas etapas, os incisivos foram novamente submetidos à ataque ácido, colagem de bráquetes ortodônticos e foram levados ao teste de cisalhamento. Com isso, pode-se avaliar a resistência adesiva nos diferentes grupos, possibilitando a observação do método que provoca maior resistência ao cisalhamento. Foram submetidos ao teste estatístico de Kruskal-Wallis que indicou não haver diferença estatística entre os grupos avaliados ( $\mathrm{p}=0,2383)$.

Palavras-chave: Esmalte dentário. Braquetes ortodônticos. Resistência ao cisalhamento.

\begin{abstract}
Proper selection of instrumental is essential for efficient removal of residual resin enamel, which can positively influence the bond strength of orthodontic brackets after rebonding. To evaluate different protocols for removal of residual resin enamel in order to maintain an adequate bond strength of orthodontic brackets in rebonding. A total of 40 bovine incisors were divided into 4 groups (G1, G2, G3 and G4), with 10 teeth each. Was made a resin matrix after conditioning with phosphoric acid for each specimen to simulate the adhesive remaining on tooth enamel, then was made to remove this remaining as the proposed protocols. The teeth were worked with the G1 monoangulate CVD tip. The teeth of G2 were worked with carbide bur low speed. The teeth were worked with the G3 high speed carbide bur. The teeth of the G4 were worked with diamond drill. After these steps, the incisors were again subjected to acid etching, bonding orthodontic brackets and were taken to shear stress. Thus, one can evaluate the bond strength in the different groups, allowing visualization method that causes greater resistance to shear. Were subjected to statistical test of Kruskal-Wallis indicated that no statistical difference among the groups $(\mathrm{p}=0.2383)$.
\end{abstract}

Keyword: Dental enamel. Orthodontic Brackets. Shear Strength.

\footnotetext{
${ }^{1}$ Cirurgião-dentista.

${ }^{2}$ Professor (a) Doutor (a), Faculdade de Odontologia, Universidade Federal do Pará.
} 


\section{INTRODUÇÃO}

A técnica mais eficiente para estabilizar a ligação com o esmalte é a "etch \& rinse" (ataque ácido e lavagem). O condicionamento ácido transforma a superfície lisa do esmalte em rugosa, através da dissolução seletiva dos cristais de hidroxiapatita, aumentando a superfície de contato do material restaurador com a superfície do dente, e a atração capilar ajuda na formação de dois tipos de "tags" de resina, os "macrotags" que são prolongamentos de resina fotopolimerizados dentro de microporosidades formadas pelos espaços que cercam os cristais de hidroxiapatita e os "microtags", pequenos prolongamentos de resina dentro das microporosidades formadas pelos núcleos dos cristais ${ }^{1}$.

Oliver $^{2}$, afirmava que falhas na remoção de todos os compósitos da superfície do esmalte, após a descolagem, resultava numa "depressão em superfície plana" que não refletia com o mesmo grau que o esmalte normal. Infelizmente, os métodos de remoção dos bráquetes que apresentavam mínimo risco de danos para o esmalte também tendiam a deixar muito residual de resina.

A queda de bráquetes, consequência de fatores como falhas na técnica de colagem, pouca retentividade de determinadas bases de bráquetes, ação da força mastigatória ${ }^{3}$ e a redução do tamanho das bases dos bráquetes por motivos estéticos ${ }^{4}$, constitui um problema rotineiro na clínica ortodôntica e resulta em atrasos no atendimento e aumento do custo da manutenção do aparelho ortodôntico fixo ${ }^{5}$.

Segundo Romano et al. ${ }^{6}$ a eficiência profissional é cada vez mais considerada no estabelecimento da relação custo benefício, e no cálculo do valor da hora despendido para realizar os procedimentos. Bishara et al. ${ }^{7}$ avaliaram o efeito de colagens repetidas utilizando o sistema adesivo Transbond XT, por meio do teste de resistência ao cisalhamento. Nessa pesquisa foram utilizados quinze molares humanos, nos quais foram inicialmente colados bráquetes de incisivo central superior (Victory Series-Unitek), utilizando o sistema adesivo Transbond XT. Para determinar a resistência ao cisalhamento foi utilizada a máquina de ensaio universal
Swick. O processo de colagem e descolagem foi repetido três vezes para cada dente, 30 minutos após a colagem, sempre utilizando braquetes novos a cada recolagem. Após cada descolagem, a resina remanescente do esmalte foi avaliada com um aumento de dez vezes e removida com uma broca carbide 279 . Os resultados da análise de variância, comparando os três grupos experimentais, indicaram ausência de diferença significativa entre os grupos. Quando foram avaliadas as mudanças entre as sequências de descolagem, a primeira e a segunda descolagem indicaram que 11 dentes tiveram uma diminuição significativa na resistência ao cisalhamento, embora quatro dentes apresentassem um aumento da resistência ao cisalhamento, que não foi estatisticamente significativo. Entre as descolagens $2 \mathrm{e}$ 3 , oito dentes tiveram um decréscimo estatisticamente significativo, apesar de 7 dentes apresentarem um aumento significativo estatisticamente na resistência ao cisalhamento. Quando foram avaliadas as descolagens 1 e 3, dez dentes tiveram um decréscimo significativo, enquanto que cinco dentes tiveram um aumento significativo na resistência ao cisalhamento. Diante dos resultados, os autores concluíram que, em geral, o maior valor obtido pelo teste de resistência ao cisalhamento foi após a colagem inicial; os resultados após a recolagem demonstraram uma resistência significativamente menor e inconsistente, pois a resistência pode tanto diminuir quanto aumentar após a segunda colagem. As alterações nos valores do teste de resistência ao cisalhamento podem ser devido às mudanças nas características morfológicas da superfície do esmalte condicionado, como um resultado da presença de resina remanescente.

Há muitos materiais e técnicas para descolagem de bráquetes e remoção dos resíduos de resinas dos dentes, mas nenhum consegue devolver a mesma lisura e brilho que o esmalte apresentava antes da colocação do aparelho ortodôntico. O sistema CVDentus, kit de fresas diamantadas para ultrassom desenvolvida pela Cloro Vale (São José dos Campos, SP, BR) visa a obter este resultado ${ }^{8}$.

A preocupação do ortodontista na etapa da descolagem de bráquetes é remover bráquete e resina remanescente sem causar danos ao esmalte. Inúmeros métodos têm sido elaborados e grande variedade de 
instrumentos tem sido oferecida pelo mercado, sem esclarecer o efeito produzido sobre o esmalte ${ }^{8,9}$.

Existem atualmente 2 classes de pontas diamantadas que podem ser utilizadas para preparo direto sobre esmalte e/ou dentina: as pontas multilaminadas rotatórias para alta-rotação e as pontas CVDentus para ultrassom. As pontas CVD são revestidas por diamante e associadas a aparelhos ultrassônicos, desgastam a dentina e o esmalte por vibração e não por rotação, o corte é mais lento, porém mais preciso, gera menos calor, menor sensibilidade e ruído muito menor, sendo mais confortável para o paciente. Além disso, apresentam maior durabilidade em relação às brocas convencionais. As pontas CVD produzem menos smear layer e estrias no preparo do que as pontas diamantadas convencionais, $\mathrm{e}$ isso poderia facilitar o condicionamento do esmalte ${ }^{10}$.

As alterações no esmalte causadas por instrumentos rotatórios podem ser irreversíveis ${ }^{11}$ e ocasionalmente remanescentes residuais podem ser encontrados na superfície do esmalte após 30 segundos de polimento ${ }^{12}$. Ao longo da introdução de novas técnicas, o design de brocas foi sendo modificado e melhorado para ser menos agressivo ao esmalte ${ }^{13}$.

Gandini Junior et al. ${ }^{14}$ estudaram diferentes métodos de remoção da resina remanescente em esmalte dentário, após descolagem de bráquetes ortodônticos, e concluíram que o método da Broca multilaminada 30 lâminas foi o que apresentou melhor resultado, contudo, causou também arranhões na superfície do esmalte remanescente.

Rossi et al. ${ }^{15}$ compararam o sistema CVDentus ao sistema de fresa multilaminada na remoção de resíduos do esmalte após a descolagem de bráquetes. Foi constatado que as superfícies analisadas demonstraram maior quantidade de riscos com o uso da fresa multilaminada, e os grupos submetidos ao emprego das pontas CVDs apresentaram menor quantidade de riscos distribuídos em uma única direção. As pontas CVDs foram eficientes na remoção da resina residual após a remoção do bráquete, produzindo pequenas alterações na superfície do esmalte.

Moreira Júnior ${ }^{16}$ fez uma análise comparativa da dentina após preparos com fresas diamantadas e ponta ultrassônica CVD, associados a condicionamento ácido em concentrações diferentes. No grupo 1 (controle) foi realizado o preparo com fresa diamantada e ponta CVD, sem tratamento da superfície dentária. No grupo 2 foi realizado o condicionamento do esmalte com ácido fosfórico a $37 \%$ e no grupo 3 o condicionamento com ácido fosfórico a $15 \%$, ambos associados aos dois tipos de preparo. Cada grupo era composto por 10 amostras de dentes bovinos. Foi utilizada nesse estudo uma ponta CVD para 10 preparos, enquanto que para a fresa convencional foi utilizado 1 fresa para 5 preparos. As amostras foram analisadas em microscópio eletrônico de varredura, para verificar a quantidade de smear layer formada nos túbulos dentinários. Foi verificado que houve diferença estatística entre os dois sistemas, sugerindo que as pontas ultrassônicas CVD proporcionaram menor quantidade de smear layer nos três grupos, e também apresentou a maior quantidade de túbulos dentinários desobstruídos nos três grupos, o que pode influenciar na seleção do sistema adesivo a ser utilizado.

A broca carbide de tungstênio tem sido o instrumento mais recomendado para remoção do remanescente adesivo tanto em baixa rotação como em alta rotação ${ }^{17}$.

Macieski et al. ${ }^{18}$ realizaram em estudo sobre os efeitos de três métodos de remoção de resina remanescente do bráquete na superfície do esmalte e observaram que a broca carbide multilaminada, em baixa rotação, removeu de forma eficiente a resina remanescente, gerando estrias leves e finas na superfície dentária, mas deixou a superfície com características do esmalte prévio à colagem do bráquete. Já a broca multilaminada, em alta rotação, removeu facilmente a resina remanescente, mas mesmo utilizada com extremo cuidado observaram-se estrias de moderadas a grandes números na avaliação microscópica. É, portanto, muito difícil remover a resina sem que se cause um efeito irreversível no esmalte.

O objetivo deste estudo foi avaliar a resistência adesiva da recolagem de bráquetes ortodônticos no esmalte, após diferentes protocolos de remoção de resina residual, por meio do teste de cisalhamento. 


\section{Materiais e Métodos}

Este estudo cumpriu integralmente os requisitos éticos de pesquisa preconizados pela Resolução 466/12 do Ministério da Saúde.

Foram utilizados 40 incisivos inferiores bovinos para confecção dos corpos de prova. Estes foram divididos aleatoriamente em 4 grupos de 10 espécimes, conforme determinações do estudo piloto e cálculo do erro amostral.

Os dentes bovinos após serem extraídos, foram limpos de restos teciduais com escovação abundante e água corrente. Foram então imersos em solução fisiológica a 0,9\% (Farmax, São Paulo, SP, BR) e armazenados em estufa biológica a $37^{\circ} \mathrm{C}$, cedida pelo Laboratório de Microbiologia da Faculdade de Engenharia de Alimentos da UFPA.

Foi utilizada uma matriz de acrílico préconfeccionada em forma da letra $\mathrm{F}$, no intuito de padronizar a inclinação de $90^{\circ}$ da face vestibular do dente onde foi colado o bráquete (Figura 1A). Os corpos de prova foram posicionados com a borda incisal em uma marcação na haste superior do acrílico e sua angulação era ajustada com cola quente na lingual fixando-se a haste inferior do acrílico (Figura 1B). Os dentes foram então fixados em tubos PVC de $25 \mathrm{~mm}$ de diâmetro (Tigre, Joinville, SC, BR) com gesso pedra tipo III (Vigodent, Rio de Janeiro, RJ, BR). Figura 1C.
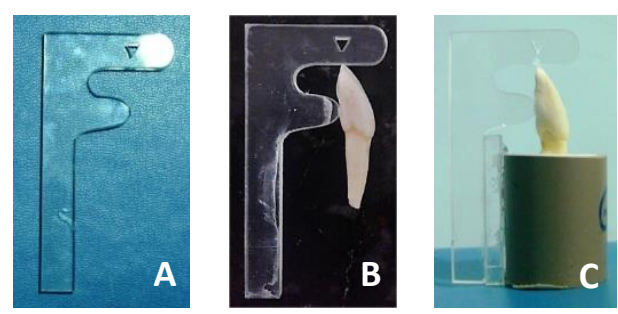

Figura 1. Matriz em acrílico (A); Corpo de prova em posição na matriz de acrílico (B) e Fixação do corpo de prova (C).

Nos 40 espécimes dos grupos foi realizada, na face vestibular, uma profilaxia com água e pedra pomes com o auxílio de escova de Robson (KG Sorensen, Cotia, SP, BR) durante 20 segundos em cada dente, e logo após isto, foi aplicado um sistema de condicionamento ácido (ácido fosfórico 37\%), (Figura 2 A), por 30 segundos. Após esse tempo foram lavados e em seguida secos com jatos de ar por 5 segundos. Ao final, foi feita a inserção de resina OrthoCem (FGM, Joinville, SC, BR), (Figura 2B), em uma matriz de acetato (Figura 2C) préconfeccionada com $4 \times 4 \mathrm{~mm}$ de largura por $1 \mathrm{~mm}$ de espessura, na face vestibular do dente e fotopolimerizada por $1 \mathrm{~min}$, para simular um remanescente de resina deixado pela descolagem de um bráquete ortodôntico.
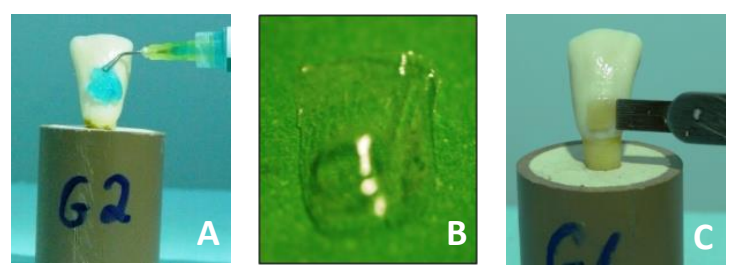

Figura 2. Condicionamento ácido (A); Inserção de incremento de resina composta (B) e Matriz de acetato (C).

Essa matriz foi confeccionada manualmente em cima de uma restauração e plastificada em máquina de plastificação de coroas (Essence Dental, São Paulo, SP, $\mathrm{BR}$ ), a fim de promover uma padronização da inserção e quantidade de resina no local, ultrapassando os limites da base do bráquete que foi colado após remoção dos resíduos de esmalte.

O tampão de resina confeccionado com matriz foi removido inicialmente com alicate de saca resina - 193 (Vision, Campinas, SP, BR) haja vista que este foi confeccionado apenas para certificação de que todo o espaço onde o bráquete foi colado havia sido preenchido por resina, evitando assim resultados falsos. Após esta etapa, foi feita a remoção minuciosa dos resíduos do esmalte de acordo com as predefinições de cada grupo, onde foram colados os bráquetes ortodônticos metálicos straight wire (Morelli Ortodontia, Sorocaba, SP, BR) de pré-molares, afim de serem submetidos ao teste de cisalhamento.

Os dentes do Grupo 1 foram trabalhados com ponta CVD uniangulada TOf $\left(\right.$ CVDentus $\left.^{\circledR}\right)$ acoplada em Ultrassom Scaler Jet D700 (Dabi Atlante, Ribeirão Preto, SP, BR) regulado na potência 4. Os dentes do Grupo 2 foram trabalhados com broca carbide de baixa rotação CA56 (Microdont, São Paulo, SP, BR). Os do 
Grupo 3 com broca carbide alta rotação 30 lâminas ff 9714 (Jet Carbide Burs, São Paulo, SP, BR) e os do Grupo 4 com ponta diamantada $1112 \mathrm{ff}\left(\right.$ KG Sorensen $\left.{ }^{\circledR}\right)$. Foi utilizada uma ponta CVD para o Grupo 1, uma broca carbide de baixa rotação para o Grupo 2, uma broca carbide alta rotação para o Grupo 3 e uma ponta diamantada em alta rotação para o Grupo 4.

Após a remoção dos resíduos do esmalte, foi realizado novo ataque ácido por 30 segundos, lavagem e secagem com jato de ar por 5 segundos. Foi inserida a resina em toda a base do bráquete, e posicionado no local preparado com auxílio de pinça porta-bráquete e fotopolimerizada por 10 segundos, em cada face ao redor do bráquete (Emitter G-Schuster $/ 1250 \mathrm{~mW} / \mathrm{cm}^{2}$, Santa Maria, RS, BR).

Os 40 espécimes dos grupos foram então levados à Máquina de Ensaio Universal (Kratos, Cotia, SP, BR), do Laboratório de Biomateriais da Faculdade de Odontologia da UFPA, e submetidos ao teste de resistência ao cisalhamento a uma velocidade de 0,05 $\mathrm{mm}$ por minuto, resistência essa avaliada em Quilogramas-força (Kgf).

A haste metálica da máquina de Teste Universal que foi confeccionada com o formato padrão do bráquete foi posicionada sobre o mesmo, no sentido incisocervical, a qual foi submetida à carga vertical de cisalhamento até ocorrer o descolamento dos bráquetes dos 40 espécimes e posterior avaliação dos resultados (Figura 3).

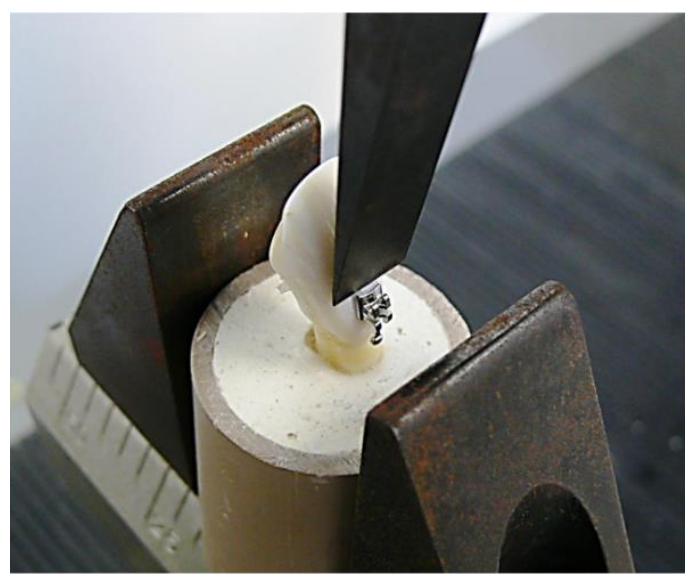

Figura 3. Teste de Cisalhamento em um dos corpos de prova.
Os dados obtidos no teste de cisalhamento foram tabulados no Microsoft Excel ${ }^{\circledR}$ e trabalhados para análise estatística inferencial através do software Bioestat 5.2.

\section{Resultados}

A Tabela 1 mostra os resultados de estatística descritiva obtida nos diferentes grupos. Os dados foram submetidos ao teste de normalidade de KolmogorovSmirnov, não obtendo normalidade. Devido os dados não serem paramétricos, foram submetidos ao teste estatístico de Kruskal-Wallis, que indicou não haver diferença estatística entre os grupos avaliados $(\mathrm{p}=0,2383)$.

Tabela 1. Resistência ao Cisalhamento dos grupos testados.

\begin{tabular}{lcccc}
\hline & G1 & G2 & G3 & G4 \\
\hline Média & 21,5 & 17,9 & 16,4 & 17,3 \\
Mínimo & 9,12 & 9,12 & 7,45 & 9,47 \\
Máximo & 30,71 & 33,77 & 23,07 & 30,52 \\
Desvio Padrão & $\pm 5,97$ & $\pm 8,19$ & $\pm 5,92$ & $\pm 5,78$ \\
\hline
\end{tabular}

Fonte: Autor da Pesquisa (junho de 2013).

O Gráfico 1 (Box-plot) detalha os resultados obtidos, no qual estão representados os valores da Tabela 1 (Média, Desvio padrão, Valores Máximos e Mínimos)

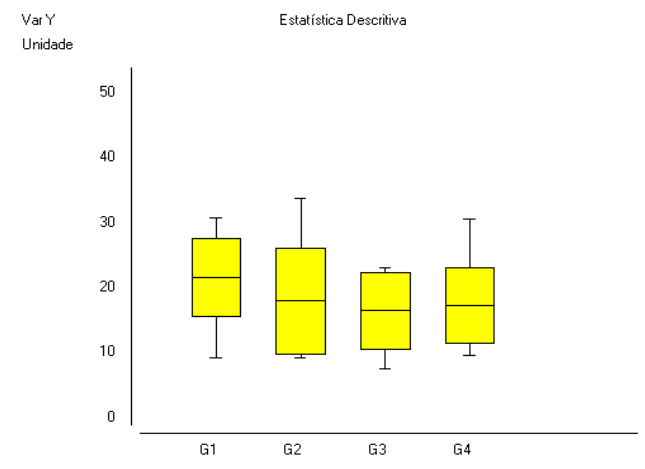

Gráfico 1. Box-plot indicando a resistência ao cisalhamento dos grupos testados. 


\section{DiscuSSÃo}

De acordo com Brown e David ${ }^{19}$, a perda de esmalte durante a remoção do bráquete e da resina remanescente tem sido relatada na literatura variando de 27,5 a $48 \mu \mathrm{m}$, $55,6 \mu \mathrm{m}$ e 26,1 a $41,2 \mu \mathrm{m}$, dependendo da quantidade de carga do material de colagem, do instrumento utilizado e da técnica de mensuração empregada, sendo clinicamente insignificante, se comparada com a espessura média do esmalte, que é de 1500 a $2000 \mu \mathrm{m}$. A quantidade de perda de esmalte durante o procedimento de descolagem torna-se clinicamente significativa quando se considera que a maior concentração de flúor está presente na superfície, e diminui rapidamente nos primeiros $20 \mu \mathrm{m}$ de esmalte.

Em um estudo feito em dentes humanos, Bishara et al. ${ }^{7}$ concluíram que em geral, o maior valor obtido pelo teste de resistência ao cisalhamento foi após a colagem inicial; os resultados após a recolagem demonstraram uma resistência significativamente menor e inconsistente, ou seja, a resistência pode tanto diminuir quanto aumentar após a segunda colagem. Consideraram ainda que as alterações nos valores do teste de resistência ao cisalhamento podem ser devido às mudanças nas características morfológicas da superfície do esmalte condicionado, como um resultado da presença de resina remanescente.

Gandini $\mathrm{Jr}$ et al. ${ }^{14}$ estudaram diferentes métodos de remoção da resina remanescente em esmalte dentários após descolagem de bráquetes ortodônticos. Concluíram que o método broca multilaminada 30 lâminas foi o que apresentou melhor resultado, contudo, o método causou também arranhões na superfície do esmalte remanescente.

Rossi et al. ${ }^{9}$ compararam o sistema CVDentus ao sistema de fresa multilaminada, na remoção de resíduos do esmalte após a descolagem de bráquetes. Foi constatado que as superfícies analisadas demonstraram maior quantidade de riscos com o uso da fresa multilaminada, enquanto os grupos submetidos ao emprego das pontas CVDs apresentaram menor quantidade de riscos, distribuídos em uma única direção. As pontas CVDs foram eficientes na remoção da resina residual após a remoção do bráquete, produzindo pequenas alterações na superfície do esmalte.

Para Oesterle et al. ${ }^{20}$ a utilização de dentes bovinos é uma alternativa viável para estudos de adesão, apesar do dente bovino permanente ou decíduo oferecer uma resistência menor que o esmalte humano, entre 21 a $44 \%$. Isso ocorre devido ao dente bovino se desenvolver mais rapidamente, possuir maiores irregularidades de superfície e apresentar os cristais do esmalte maiores. Entretanto, as vantagens de utilização desses dentes suprem essas desvantagens, pois apresentam estruturas semelhantes às do esmalte humano e maior facilidade de aquisição.

Romano et al. ${ }^{6}$ compararam a resistência ao cisalhamento de bráquetes metálicos fixados em esmalte bovino e humano e observaram o Índice de Remanescente Adesivo (IRA). A amostra constituída de 30 dentes, divididos em 3 grupos, GI incisivo inferior permanente humano; GII incisivo inferior permanente bovino e GIII incisivo inferior decíduo bovino. Os autores concluíram que não foi observada diferença estatisticamente significativa entre os grupos, tanto nos valores de resistência ao cisalhamento quanto na avaliação do IRA.

Silva ${ }^{21}$, em 1995, realizou um estudo que testou o tipo de dente (humano, bovino e suíno), o período de armazenamento e a superfície (dentina e esmalte), aplicando o teste de cisalhamento na máquina Universal Kratus. De acordo com a metodologia empregada e a análise estatística dos resultados registrados, concluiu que em relação ao esmalte, os dentes humanos e bovinos não apresentaram diferença estatística significante em adesão, assim como não houve também diferença estatística significante em dentina, provando que o dente bovino pode ser bem empregado em ensaios de cisalhamento como substitutos dos dentes humanos.

Giannini et $\mathrm{al}^{22}$ avaliaram a resistência adesiva de dois cimentos utilizados na colagem de acessórios ortodônticos ao esmalte dentário bovino, sendo um de polimerização química (Concise Ortodôntico ${ }^{\circledR}-3 \mathrm{M}$ Unitek) e outro fotopolimerizável (Transbond $\mathrm{XT}^{\circledR}-3 \mathrm{M}$ Unitek), e concluíram que os dentes colados com Transbond, mantiveram-se com um resultado mais uniforme entre as cargas submetidas, já os dentes 
colados com Concise, mostraram-se menos sensíveis às diferentes forças utilizadas. Não houve diferença estatística entre as cargas em um único grupo, porém, o grupo tratado com a resina Concise revelou resultados maiores ao cisalhamento, obtendo resultados com diferença estatística. Não houve em nenhum dos grupos fratura entre a interface bráquete/cimento.

Pithon et al. $^{23}$ compararam a resistência ao cisalhamento de bráquetes metálicos colados com compósito Right-on (TP Ortho, Tokyo, JP) em diferentes condições de superfícies. Utilizaram 45 incisivos inferiores permanentes bovinos divididos em 3 grupos: GI controle (transbond tx); GII right-on (ativador tanto no bráquete quanto no esmalte) e GIII right-on (líquido ativador somente na interface esmalte). Concluíram que entre os grupos GI e GII não houve diferença estatisticamente significante, já no grupo GIII houve uma diminuição da resistência ao cisalhamento, com diferença estatisticamente significante, quando comparado aos grupos GI e GII. Em todos os grupos, a maioria das fraturas ocorreu na interface bráquete/compósito, restando o material aderido no esmalte.

Tortamano et al. ${ }^{24}$ tiveram por objetivo avaliar a força de tração em bráquetes colados pela técnica indireta, com diferentes sistemas de adesão. Concluíram que nos grupos G3 e G4, (colagem indireta dos bráquetes) obtiveram uma menor resistência à tração do que nos grupos G1 e G2 (colagem direta de bráquetes). A remoção de bráquete e da resina é facilitada ao final do tratamento ortodôntico quando os bráquetes são colados pela técnica indireta, pois a interface criada entre a resina polimerizada e o adesivo torna-se a linha de fratura no momento da remoção do bráquete ${ }^{25}$. Hocevar et al. ${ }^{26}$ realizaram um estudo em que a linha de fratura entre o esmalte e a resina ocorreu em $72 \%$ dos casos em que se utilizou a colagem indireta, e 56\% naqueles em que a colagem direta foi utilizada.

\section{Conclusão}

De acordo com a metodologia utilizada e com base nos resultados obtidos, conclui-se que os diferentes protocolos de remoção de resina residual não influenciaram na resistência adesiva da recolagem de bráquetes ortodônticos $(\mathrm{p}=0,2383)$.

\section{REFERÊNCIAS}

1. Van Meerbeek B, De Munck J, Yoshida Y, Inoue S, Vargas M, Vijay P, et al. Buonocore memorial lecture. Adhesion to enamel and dentin: current status and future challenges. Oper Dent. 2003 fev; 28(3):215-35.

2. Oliver RG. A new instrument for debonding clean-up. $J$ Clin Orthod. 1991; 25:407-10.

3. Oesterle LJ, Shellhart WC, Belanger HK. The use of bovine enamel in bonding studies. Am J Orthod Dentofacial Orthop. 1998; 114(5):514-9.

4. MacColl GA, Rossouw PE, Titley KC, Yamin C. The relationship between bond strength and orthodontic bracket base surface area with conventional and microetched foil-mesh bases. Am J Orthod Dentofacial Orthop. 1998; 113(3):276-81.

5. Lunardi N. Análise in vitro da resistência ao cisalhamento de bráquetes metálicos submetidos à reciclagem repetida, fixados com diferentes materiais. Piracicaba (SP): Faculdade de Odontologia de Piracicaba. 2004.

6. Romano FL, Tavares SW, Ramalli EL, Magnani MBBA, Nouer DF. Análise in vitro da resistência ao cisalhamento de braquetes metálicos colados em incisivos bovinos e humanos. $R$ Dental Press Ortodon Ortop Facial. 2004 nov/dez; 9(6):63-9.

7. Bishara SE, VonWald L, Laffoon JF, Warren JJ. The effect of repeated bonding on the shear bond strength of a composite resin orthodontic adhesive. Angle Orthod. 2000; 70(6):435-41.

8. Santos-Pinto A, et al. Remoção de resina residual do esmalte dentário após descolagem de acessórios ortodônticos: avaliação de duas técnicas. Rev Gaúcha de Ortodontia. 2001; 5:42-8.

9. Rossi TC, Santos-Pinto L, Santos-Pinto A, Lima LM. Eficiência do sistema CVDentus e fresas multilaminadas na remoção de resíduos do esmalte após descolagem de bráquetes. Braz Oral Res. 2004; 18 Suppl Proceedings of the 21nd annual SBPqO Meeting: 68.

10. Vieira D, Vieira D. Pontas de diamante CVD: início ou fim da alta rotação? JADA Brasil. 2002 set/out; 5(10): 307-13. 
11. Piacentini C, Sfondrini, G. A scanning electron microscopy comparison of enamel polishing methods after air-rotor stripping. Am J Orthod Dentofacial Orthop. 1996 jan; 109(1): 7-63.

12. Vieira AC, Pinto RA, Chevitarese O, et al. Polishing after debracketing: its influence upon enamel surface. $J$ Clin Pediatr Dent. 1993; 18(1):7-11.

13. Radlanski RJ. A new carbide finishing bur for bracket debonding. J Orofac Orthop. 2001 jul;62 (4):296-304.

14. Gandini Junior LG, et al. Avaliação de diferentes métodos de remoção da resina remanescente ao esmalte dentário após descolagem de bráquetes ortodônticos. Ortodontia. 1995; 28: 53-60.

15. Rossi TC, Santos-Pinto L, Santos-Pinto A, Lima LM. Eficiência do sistema CVDentus e fresas multilaminadas na remoção de resíduos do esmalte após descolagem de bráquetes. Braz Oral Res. 2004; 18 Suppl Proceedings of the 21nd annual SBPqO Meeting: 68.

16. Moreira Junior MT. Análise por microscopia eletrônica de varredura da "smear layer" produzida em preparos com pontas diamantadas e pontas CVD. Dissertação (Mestrado em Odontologia). Faculdade de Odontologia. Universidade Federal do Pará, Belém, PA; 2008. 73 p.

17. Campbell PM. Enamel surfaces after orthodontic braquetes the bonding. Angle Orthod. 1995; 65(2):10310 .

18. Macieski K, Rocha R, Locks Arno, Ribeiro Gerson U. Avaliação dos três métodos de remoção de resina remanescente do bráquete na superfície do esmalte. Dental Press J Orthodontic. 2011 sept/oct; 16(5):146-54

19. Brown CRL, David CW. Enamel loss during orthodontic bonding and subsequent loss during removal of filled and unfiled adhesives. Am J Orthodontic. 1978; 74(6):663-71.

20. Oesterle LJ, Shellhart WC, Belanger HK. The use of bovine enamel in bonding studies. Am J Orthod Dentofacial Orthop. 1998; 114(5):514-9.

21. Silva CM. Avaliação in vitro da resistência ao cisalhamento de um sistema adesivo em esmalte e dentina de dentes humanos bovinos e suínos. Dissertação (Mestrado em Dentística). Faculdade de Odontologia. Universidade de São Paulo. Bauru; 1995. 76p.

22. Giannini C, Francisconi PAS. Resistência a remoção de braquetes ortodônticos sob ação de diferentes cargas contínuas. R Dental Press Ortodon Ortop Facial. 2008 maio/jun; 13 (3): 50-9.

23. Pithon MM, et al. Avaliação da resistência ao cisalhamento do compósito right-on em diferentes condições de esmalte. $R$ Dental Press Ortodon Ortop Facial. 2008 maio/jun; 13(3):60-5.

24. Tortamano A et al. Avaliação da força de tração em braquetes colados pela técnica indireta com sistema de adesão. R Dental Press Ortodon Ortop Facial. 2007 maio/jun; 12 (3):104-10.
25. Thomas RG. Indirect bonding/ simplicity in action. $J$ Clin Orthod. 1979; 13:93-105.

26. Hocevar RA, Vicent HF. Indirect versus direct Bond ing: Bond and failure location. Am J Orthod Dentofacial Orthop. 1988; 94: 367-71. 